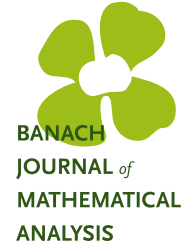

Banach J. Math. Anal. 10 (2016), no. 3, 608-620

http://dx.doi.org/10.1215/17358787-3607486

ISSN: $1735-8787$ (electronic)

http://projecteuclid.org/bjma

\title{
VECTOR-VALUED CHARACTERS ON VECTOR-VALUED FUNCTION ALGEBRAS
}

\author{
MORTAZA ABTAHI
}

Communicated by K. Jarosz

\begin{abstract}
Let $A$ be a commutative unital Banach algebra and let $X$ be a compact space. We study the class of $A$-valued function algebras on $X$ as subalgebras of $C(X, A)$ with certain properties. We introduce the notion of $A$-characters of an $A$-valued function algebra $\mathscr{A}$ as homomorphisms from $\mathscr{A}$ into $A$ that basically have the same properties as evaluation homomorphisms $\mathcal{E}_{x}: f \mapsto f(x)$, with $x \in X$. We show that, under certain conditions, there is a one-to-one correspondence between the set of $A$-characters of $\mathscr{A}$ and the set of characters of the function algebra $\mathfrak{A}=\mathscr{A} \cap C(X)$ of all scalar-valued functions in $\mathscr{A}$. For the so-called natural $A$-valued function algebras, such as $C(X, A)$ and $\operatorname{Lip}(X, A)$, we show that $\mathcal{E}_{x}(x \in X)$ are the only $A$-characters. Vector-valued characters are utilized to identify vector-valued spectra.
\end{abstract}

\section{INTRODUCTION AND PRELIMINARIES}

In this article, we consider only commutative unital Banach algebras over the complex field $\mathbb{C}$ (see [3], [4], [11], [18]).

Let $A$ be a commutative Banach algebra. The set of all characters of $A$ is denoted by $\mathfrak{M}(A)$. It is well known that $\mathfrak{M}(A)$, equipped with the Gelfand topology, is a compact Hausdorff space called the character space of $A$. For every $a \in A$, let $\hat{a}: \mathfrak{M}(A) \rightarrow \mathbb{C}, \phi \mapsto \phi(a)$, be the Gelfand transform of $a$. The algebra $A$ then can be seen, through its Gelfand representation $A \rightarrow C(\mathfrak{M}(A)), a \mapsto \hat{a}$, as a subalgebra of $C(\mathfrak{M}(A))$.

Copyright 2016 by the Tusi Mathematical Research Group.

Received May 23, 2015; Accepted Nov. 27, 2015.

2010 Mathematics Subject Classification. Primary 46J10; Secondary 46J20, 46H10, 46E40.

Keywords. Algebras of continuous vector-valued functions, Banach function algebras, vectorvalued function algebras, characters, maximal ideals. 


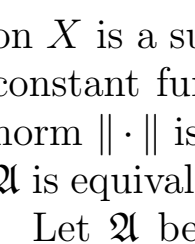

Banach J. Math. Anal. 10 (2016), no. 3, 608-620

http://dx.doi.org/10.1215/17358787-3607486

ISSN: $1735-8787$ (electronic)

http://projecteuclid.org/bjma

\title{
VECTOR-VALUED CHARACTERS ON VECTOR-VALUED FUNCTION ALGEBRAS
}

\author{
MORTAZA ABTAHI
}

Communicated by K. Jarosz

\begin{abstract}
Let $A$ be a commutative unital Banach algebra and let $X$ be a compact space. We study the class of $A$-valued function algebras on $X$ as subalgebras of $C(X, A)$ with certain properties. We introduce the notion of $A$-characters of an $A$-valued function algebra $\mathscr{A}$ as homomorphisms from $\mathscr{A}$ into $A$ that basically have the same properties as evaluation homomorphisms $\mathcal{E}_{x}: f \mapsto f(x)$, with $x \in X$. We show that, under certain conditions, there is a one-to-one correspondence between the set of $A$-characters of $\mathscr{A}$ and the set of characters of the function algebra $\mathfrak{A}=\mathscr{A} \cap C(X)$ of all scalar-valued functions in $\mathscr{A}$. For the so-called natural $A$-valued function algebras, such as $C(X, A)$ and $\operatorname{Lip}(X, A)$, we show that $\mathcal{E}_{x}(x \in X)$ are the only $A$-characters. Vector-valued characters are utilized to identify vector-valued spectra.
\end{abstract}

\section{INTRODUCTION AND PRELIMINARIES}

In this article, we consider only commutative unital Banach algebras over the complex field $\mathbb{C}$ (see [3], [4], [11], [18]).

Let $A$ be a commutative Banach algebra. The set of all characters of $A$ is denoted by $\mathfrak{M}(A)$. It is well known that $\mathfrak{M}(A)$, equipped with the Gelfand topology, is a compact Hausdorff space called the character space of $A$. For every $a \in A$, let $\hat{a}: \mathfrak{M}(A) \rightarrow \mathbb{C}, \phi \mapsto \phi(a)$, be the Gelfand transform of $a$. The algebra $A$ then can be seen, through its Gelfand representation $A \rightarrow C(\mathfrak{M}(A)), a \mapsto \hat{a}$, as a subalgebra of $C(\mathfrak{M}(A))$.

Copyright 2016 by the Tusi Mathematical Research Group.

Received May 23, 2015; Accepted Nov. 27, 2015.

2010 Mathematics Subject Classification. Primary 46J10; Secondary 46J20, 46H10, 46E40.

Keywords. Algebras of continuous vector-valued functions, Banach function algebras, vectorvalued function algebras, characters, maximal ideals. 


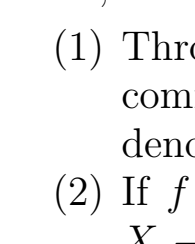

Banach J. Math. Anal. 10 (2016), no. 3, 608-620

http://dx.doi.org/10.1215/17358787-3607486

ISSN: $1735-8787$ (electronic)

http://projecteuclid.org/bjma

\title{
VECTOR-VALUED CHARACTERS ON VECTOR-VALUED FUNCTION ALGEBRAS
}

\author{
MORTAZA ABTAHI
}

Communicated by K. Jarosz

\begin{abstract}
Let $A$ be a commutative unital Banach algebra and let $X$ be a compact space. We study the class of $A$-valued function algebras on $X$ as subalgebras of $C(X, A)$ with certain properties. We introduce the notion of $A$-characters of an $A$-valued function algebra $\mathscr{A}$ as homomorphisms from $\mathscr{A}$ into $A$ that basically have the same properties as evaluation homomorphisms $\mathcal{E}_{x}: f \mapsto f(x)$, with $x \in X$. We show that, under certain conditions, there is a one-to-one correspondence between the set of $A$-characters of $\mathscr{A}$ and the set of characters of the function algebra $\mathfrak{A}=\mathscr{A} \cap C(X)$ of all scalar-valued functions in $\mathscr{A}$. For the so-called natural $A$-valued function algebras, such as $C(X, A)$ and $\operatorname{Lip}(X, A)$, we show that $\mathcal{E}_{x}(x \in X)$ are the only $A$-characters. Vector-valued characters are utilized to identify vector-valued spectra.
\end{abstract}

\section{INTRODUCTION AND PRELIMINARIES}

In this article, we consider only commutative unital Banach algebras over the complex field $\mathbb{C}$ (see [3], [4], [11], [18]).

Let $A$ be a commutative Banach algebra. The set of all characters of $A$ is denoted by $\mathfrak{M}(A)$. It is well known that $\mathfrak{M}(A)$, equipped with the Gelfand topology, is a compact Hausdorff space called the character space of $A$. For every $a \in A$, let $\hat{a}: \mathfrak{M}(A) \rightarrow \mathbb{C}, \phi \mapsto \phi(a)$, be the Gelfand transform of $a$. The algebra $A$ then can be seen, through its Gelfand representation $A \rightarrow C(\mathfrak{M}(A)), a \mapsto \hat{a}$, as a subalgebra of $C(\mathfrak{M}(A))$.

Copyright 2016 by the Tusi Mathematical Research Group.

Received May 23, 2015; Accepted Nov. 27, 2015.

2010 Mathematics Subject Classification. Primary 46J10; Secondary 46J20, 46H10, 46E40.

Keywords. Algebras of continuous vector-valued functions, Banach function algebras, vectorvalued function algebras, characters, maximal ideals. 


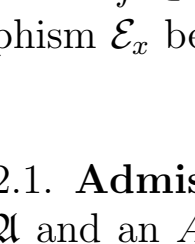

Banach J. Math. Anal. 10 (2016), no. 3, 608-620

http://dx.doi.org/10.1215/17358787-3607486

ISSN: $1735-8787$ (electronic)

http://projecteuclid.org/bjma

\title{
VECTOR-VALUED CHARACTERS ON VECTOR-VALUED FUNCTION ALGEBRAS
}

\author{
MORTAZA ABTAHI
}

Communicated by K. Jarosz

\begin{abstract}
Let $A$ be a commutative unital Banach algebra and let $X$ be a compact space. We study the class of $A$-valued function algebras on $X$ as subalgebras of $C(X, A)$ with certain properties. We introduce the notion of $A$-characters of an $A$-valued function algebra $\mathscr{A}$ as homomorphisms from $\mathscr{A}$ into $A$ that basically have the same properties as evaluation homomorphisms $\mathcal{E}_{x}: f \mapsto f(x)$, with $x \in X$. We show that, under certain conditions, there is a one-to-one correspondence between the set of $A$-characters of $\mathscr{A}$ and the set of characters of the function algebra $\mathfrak{A}=\mathscr{A} \cap C(X)$ of all scalar-valued functions in $\mathscr{A}$. For the so-called natural $A$-valued function algebras, such as $C(X, A)$ and $\operatorname{Lip}(X, A)$, we show that $\mathcal{E}_{x}(x \in X)$ are the only $A$-characters. Vector-valued characters are utilized to identify vector-valued spectra.
\end{abstract}

\section{INTRODUCTION AND PRELIMINARIES}

In this article, we consider only commutative unital Banach algebras over the complex field $\mathbb{C}$ (see [3], [4], [11], [18]).

Let $A$ be a commutative Banach algebra. The set of all characters of $A$ is denoted by $\mathfrak{M}(A)$. It is well known that $\mathfrak{M}(A)$, equipped with the Gelfand topology, is a compact Hausdorff space called the character space of $A$. For every $a \in A$, let $\hat{a}: \mathfrak{M}(A) \rightarrow \mathbb{C}, \phi \mapsto \phi(a)$, be the Gelfand transform of $a$. The algebra $A$ then can be seen, through its Gelfand representation $A \rightarrow C(\mathfrak{M}(A)), a \mapsto \hat{a}$, as a subalgebra of $C(\mathfrak{M}(A))$.

Copyright 2016 by the Tusi Mathematical Research Group.

Received May 23, 2015; Accepted Nov. 27, 2015.

2010 Mathematics Subject Classification. Primary 46J10; Secondary 46J20, 46H10, 46E40.

Keywords. Algebras of continuous vector-valued functions, Banach function algebras, vectorvalued function algebras, characters, maximal ideals. 


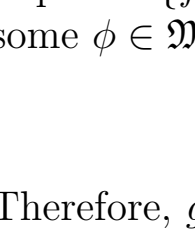

Banach J. Math. Anal. 10 (2016), no. 3, 608-620

http://dx.doi.org/10.1215/17358787-3607486

ISSN: $1735-8787$ (electronic)

http://projecteuclid.org/bjma

\title{
VECTOR-VALUED CHARACTERS ON VECTOR-VALUED FUNCTION ALGEBRAS
}

\author{
MORTAZA ABTAHI
}

Communicated by K. Jarosz

\begin{abstract}
Let $A$ be a commutative unital Banach algebra and let $X$ be a compact space. We study the class of $A$-valued function algebras on $X$ as subalgebras of $C(X, A)$ with certain properties. We introduce the notion of $A$-characters of an $A$-valued function algebra $\mathscr{A}$ as homomorphisms from $\mathscr{A}$ into $A$ that basically have the same properties as evaluation homomorphisms $\mathcal{E}_{x}: f \mapsto f(x)$, with $x \in X$. We show that, under certain conditions, there is a one-to-one correspondence between the set of $A$-characters of $\mathscr{A}$ and the set of characters of the function algebra $\mathfrak{A}=\mathscr{A} \cap C(X)$ of all scalar-valued functions in $\mathscr{A}$. For the so-called natural $A$-valued function algebras, such as $C(X, A)$ and $\operatorname{Lip}(X, A)$, we show that $\mathcal{E}_{x}(x \in X)$ are the only $A$-characters. Vector-valued characters are utilized to identify vector-valued spectra.
\end{abstract}

\section{INTRODUCTION AND PRELIMINARIES}

In this article, we consider only commutative unital Banach algebras over the complex field $\mathbb{C}$ (see [3], [4], [11], [18]).

Let $A$ be a commutative Banach algebra. The set of all characters of $A$ is denoted by $\mathfrak{M}(A)$. It is well known that $\mathfrak{M}(A)$, equipped with the Gelfand topology, is a compact Hausdorff space called the character space of $A$. For every $a \in A$, let $\hat{a}: \mathfrak{M}(A) \rightarrow \mathbb{C}, \phi \mapsto \phi(a)$, be the Gelfand transform of $a$. The algebra $A$ then can be seen, through its Gelfand representation $A \rightarrow C(\mathfrak{M}(A)), a \mapsto \hat{a}$, as a subalgebra of $C(\mathfrak{M}(A))$.

Copyright 2016 by the Tusi Mathematical Research Group.

Received May 23, 2015; Accepted Nov. 27, 2015.

2010 Mathematics Subject Classification. Primary 46J10; Secondary 46J20, 46H10, 46E40.

Keywords. Algebras of continuous vector-valued functions, Banach function algebras, vectorvalued function algebras, characters, maximal ideals. 


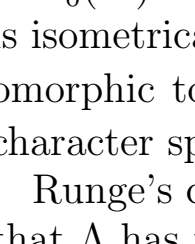

Banach J. Math. Anal. 10 (2016), no. 3, 608-620

http://dx.doi.org/10.1215/17358787-3607486

ISSN: $1735-8787$ (electronic)

http://projecteuclid.org/bjma

\title{
VECTOR-VALUED CHARACTERS ON VECTOR-VALUED FUNCTION ALGEBRAS
}

\author{
MORTAZA ABTAHI
}

Communicated by K. Jarosz

\begin{abstract}
Let $A$ be a commutative unital Banach algebra and let $X$ be a compact space. We study the class of $A$-valued function algebras on $X$ as subalgebras of $C(X, A)$ with certain properties. We introduce the notion of $A$-characters of an $A$-valued function algebra $\mathscr{A}$ as homomorphisms from $\mathscr{A}$ into $A$ that basically have the same properties as evaluation homomorphisms $\mathcal{E}_{x}: f \mapsto f(x)$, with $x \in X$. We show that, under certain conditions, there is a one-to-one correspondence between the set of $A$-characters of $\mathscr{A}$ and the set of characters of the function algebra $\mathfrak{A}=\mathscr{A} \cap C(X)$ of all scalar-valued functions in $\mathscr{A}$. For the so-called natural $A$-valued function algebras, such as $C(X, A)$ and $\operatorname{Lip}(X, A)$, we show that $\mathcal{E}_{x}(x \in X)$ are the only $A$-characters. Vector-valued characters are utilized to identify vector-valued spectra.
\end{abstract}

\section{INTRODUCTION AND PRELIMINARIES}

In this article, we consider only commutative unital Banach algebras over the complex field $\mathbb{C}$ (see [3], [4], [11], [18]).

Let $A$ be a commutative Banach algebra. The set of all characters of $A$ is denoted by $\mathfrak{M}(A)$. It is well known that $\mathfrak{M}(A)$, equipped with the Gelfand topology, is a compact Hausdorff space called the character space of $A$. For every $a \in A$, let $\hat{a}: \mathfrak{M}(A) \rightarrow \mathbb{C}, \phi \mapsto \phi(a)$, be the Gelfand transform of $a$. The algebra $A$ then can be seen, through its Gelfand representation $A \rightarrow C(\mathfrak{M}(A)), a \mapsto \hat{a}$, as a subalgebra of $C(\mathfrak{M}(A))$.

Copyright 2016 by the Tusi Mathematical Research Group.

Received May 23, 2015; Accepted Nov. 27, 2015.

2010 Mathematics Subject Classification. Primary 46J10; Secondary 46J20, 46H10, 46E40.

Keywords. Algebras of continuous vector-valued functions, Banach function algebras, vectorvalued function algebras, characters, maximal ideals. 


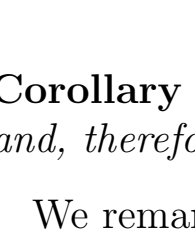

Banach J. Math. Anal. 10 (2016), no. 3, 608-620

http://dx.doi.org/10.1215/17358787-3607486

ISSN: $1735-8787$ (electronic)

http://projecteuclid.org/bjma

\title{
VECTOR-VALUED CHARACTERS ON VECTOR-VALUED FUNCTION ALGEBRAS
}

\author{
MORTAZA ABTAHI
}

Communicated by K. Jarosz

\begin{abstract}
Let $A$ be a commutative unital Banach algebra and let $X$ be a compact space. We study the class of $A$-valued function algebras on $X$ as subalgebras of $C(X, A)$ with certain properties. We introduce the notion of $A$-characters of an $A$-valued function algebra $\mathscr{A}$ as homomorphisms from $\mathscr{A}$ into $A$ that basically have the same properties as evaluation homomorphisms $\mathcal{E}_{x}: f \mapsto f(x)$, with $x \in X$. We show that, under certain conditions, there is a one-to-one correspondence between the set of $A$-characters of $\mathscr{A}$ and the set of characters of the function algebra $\mathfrak{A}=\mathscr{A} \cap C(X)$ of all scalar-valued functions in $\mathscr{A}$. For the so-called natural $A$-valued function algebras, such as $C(X, A)$ and $\operatorname{Lip}(X, A)$, we show that $\mathcal{E}_{x}(x \in X)$ are the only $A$-characters. Vector-valued characters are utilized to identify vector-valued spectra.
\end{abstract}

\section{INTRODUCTION AND PRELIMINARIES}

In this article, we consider only commutative unital Banach algebras over the complex field $\mathbb{C}$ (see [3], [4], [11], [18]).

Let $A$ be a commutative Banach algebra. The set of all characters of $A$ is denoted by $\mathfrak{M}(A)$. It is well known that $\mathfrak{M}(A)$, equipped with the Gelfand topology, is a compact Hausdorff space called the character space of $A$. For every $a \in A$, let $\hat{a}: \mathfrak{M}(A) \rightarrow \mathbb{C}, \phi \mapsto \phi(a)$, be the Gelfand transform of $a$. The algebra $A$ then can be seen, through its Gelfand representation $A \rightarrow C(\mathfrak{M}(A)), a \mapsto \hat{a}$, as a subalgebra of $C(\mathfrak{M}(A))$.

Copyright 2016 by the Tusi Mathematical Research Group.

Received May 23, 2015; Accepted Nov. 27, 2015.

2010 Mathematics Subject Classification. Primary 46J10; Secondary 46J20, 46H10, 46E40.

Keywords. Algebras of continuous vector-valued functions, Banach function algebras, vectorvalued function algebras, characters, maximal ideals. 


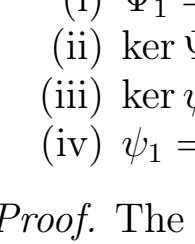

Banach J. Math. Anal. 10 (2016), no. 3, 608-620

http://dx.doi.org/10.1215/17358787-3607486

ISSN: $1735-8787$ (electronic)

http://projecteuclid.org/bjma

\title{
VECTOR-VALUED CHARACTERS ON VECTOR-VALUED FUNCTION ALGEBRAS
}

\author{
MORTAZA ABTAHI
}

Communicated by K. Jarosz

\begin{abstract}
Let $A$ be a commutative unital Banach algebra and let $X$ be a compact space. We study the class of $A$-valued function algebras on $X$ as subalgebras of $C(X, A)$ with certain properties. We introduce the notion of $A$-characters of an $A$-valued function algebra $\mathscr{A}$ as homomorphisms from $\mathscr{A}$ into $A$ that basically have the same properties as evaluation homomorphisms $\mathcal{E}_{x}: f \mapsto f(x)$, with $x \in X$. We show that, under certain conditions, there is a one-to-one correspondence between the set of $A$-characters of $\mathscr{A}$ and the set of characters of the function algebra $\mathfrak{A}=\mathscr{A} \cap C(X)$ of all scalar-valued functions in $\mathscr{A}$. For the so-called natural $A$-valued function algebras, such as $C(X, A)$ and $\operatorname{Lip}(X, A)$, we show that $\mathcal{E}_{x}(x \in X)$ are the only $A$-characters. Vector-valued characters are utilized to identify vector-valued spectra.
\end{abstract}

\section{INTRODUCTION AND PRELIMINARIES}

In this article, we consider only commutative unital Banach algebras over the complex field $\mathbb{C}$ (see [3], [4], [11], [18]).

Let $A$ be a commutative Banach algebra. The set of all characters of $A$ is denoted by $\mathfrak{M}(A)$. It is well known that $\mathfrak{M}(A)$, equipped with the Gelfand topology, is a compact Hausdorff space called the character space of $A$. For every $a \in A$, let $\hat{a}: \mathfrak{M}(A) \rightarrow \mathbb{C}, \phi \mapsto \phi(a)$, be the Gelfand transform of $a$. The algebra $A$ then can be seen, through its Gelfand representation $A \rightarrow C(\mathfrak{M}(A)), a \mapsto \hat{a}$, as a subalgebra of $C(\mathfrak{M}(A))$.

Copyright 2016 by the Tusi Mathematical Research Group.

Received May 23, 2015; Accepted Nov. 27, 2015.

2010 Mathematics Subject Classification. Primary 46J10; Secondary 46J20, 46H10, 46E40.

Keywords. Algebras of continuous vector-valued functions, Banach function algebras, vectorvalued function algebras, characters, maximal ideals. 


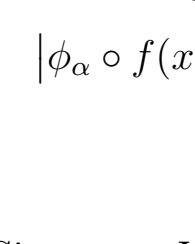

Banach J. Math. Anal. 10 (2016), no. 3, 608-620

http://dx.doi.org/10.1215/17358787-3607486

ISSN: $1735-8787$ (electronic)

http://projecteuclid.org/bjma

\title{
VECTOR-VALUED CHARACTERS ON VECTOR-VALUED FUNCTION ALGEBRAS
}

\author{
MORTAZA ABTAHI
}

Communicated by K. Jarosz

\begin{abstract}
Let $A$ be a commutative unital Banach algebra and let $X$ be a compact space. We study the class of $A$-valued function algebras on $X$ as subalgebras of $C(X, A)$ with certain properties. We introduce the notion of $A$-characters of an $A$-valued function algebra $\mathscr{A}$ as homomorphisms from $\mathscr{A}$ into $A$ that basically have the same properties as evaluation homomorphisms $\mathcal{E}_{x}: f \mapsto f(x)$, with $x \in X$. We show that, under certain conditions, there is a one-to-one correspondence between the set of $A$-characters of $\mathscr{A}$ and the set of characters of the function algebra $\mathfrak{A}=\mathscr{A} \cap C(X)$ of all scalar-valued functions in $\mathscr{A}$. For the so-called natural $A$-valued function algebras, such as $C(X, A)$ and $\operatorname{Lip}(X, A)$, we show that $\mathcal{E}_{x}(x \in X)$ are the only $A$-characters. Vector-valued characters are utilized to identify vector-valued spectra.
\end{abstract}

\section{INTRODUCTION AND PRELIMINARIES}

In this article, we consider only commutative unital Banach algebras over the complex field $\mathbb{C}$ (see [3], [4], [11], [18]).

Let $A$ be a commutative Banach algebra. The set of all characters of $A$ is denoted by $\mathfrak{M}(A)$. It is well known that $\mathfrak{M}(A)$, equipped with the Gelfand topology, is a compact Hausdorff space called the character space of $A$. For every $a \in A$, let $\hat{a}: \mathfrak{M}(A) \rightarrow \mathbb{C}, \phi \mapsto \phi(a)$, be the Gelfand transform of $a$. The algebra $A$ then can be seen, through its Gelfand representation $A \rightarrow C(\mathfrak{M}(A)), a \mapsto \hat{a}$, as a subalgebra of $C(\mathfrak{M}(A))$.

Copyright 2016 by the Tusi Mathematical Research Group.

Received May 23, 2015; Accepted Nov. 27, 2015.

2010 Mathematics Subject Classification. Primary 46J10; Secondary 46J20, 46H10, 46E40.

Keywords. Algebras of continuous vector-valued functions, Banach function algebras, vectorvalued function algebras, characters, maximal ideals. 


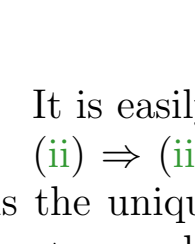

Banach J. Math. Anal. 10 (2016), no. 3, 608-620

http://dx.doi.org/10.1215/17358787-3607486

ISSN: $1735-8787$ (electronic)

http://projecteuclid.org/bjma

\title{
VECTOR-VALUED CHARACTERS ON VECTOR-VALUED FUNCTION ALGEBRAS
}

\author{
MORTAZA ABTAHI
}

Communicated by K. Jarosz

\begin{abstract}
Let $A$ be a commutative unital Banach algebra and let $X$ be a compact space. We study the class of $A$-valued function algebras on $X$ as subalgebras of $C(X, A)$ with certain properties. We introduce the notion of $A$-characters of an $A$-valued function algebra $\mathscr{A}$ as homomorphisms from $\mathscr{A}$ into $A$ that basically have the same properties as evaluation homomorphisms $\mathcal{E}_{x}: f \mapsto f(x)$, with $x \in X$. We show that, under certain conditions, there is a one-to-one correspondence between the set of $A$-characters of $\mathscr{A}$ and the set of characters of the function algebra $\mathfrak{A}=\mathscr{A} \cap C(X)$ of all scalar-valued functions in $\mathscr{A}$. For the so-called natural $A$-valued function algebras, such as $C(X, A)$ and $\operatorname{Lip}(X, A)$, we show that $\mathcal{E}_{x}(x \in X)$ are the only $A$-characters. Vector-valued characters are utilized to identify vector-valued spectra.
\end{abstract}

\section{INTRODUCTION AND PRELIMINARIES}

In this article, we consider only commutative unital Banach algebras over the complex field $\mathbb{C}$ (see [3], [4], [11], [18]).

Let $A$ be a commutative Banach algebra. The set of all characters of $A$ is denoted by $\mathfrak{M}(A)$. It is well known that $\mathfrak{M}(A)$, equipped with the Gelfand topology, is a compact Hausdorff space called the character space of $A$. For every $a \in A$, let $\hat{a}: \mathfrak{M}(A) \rightarrow \mathbb{C}, \phi \mapsto \phi(a)$, be the Gelfand transform of $a$. The algebra $A$ then can be seen, through its Gelfand representation $A \rightarrow C(\mathfrak{M}(A)), a \mapsto \hat{a}$, as a subalgebra of $C(\mathfrak{M}(A))$.

Copyright 2016 by the Tusi Mathematical Research Group.

Received May 23, 2015; Accepted Nov. 27, 2015.

2010 Mathematics Subject Classification. Primary 46J10; Secondary 46J20, 46H10, 46E40.

Keywords. Algebras of continuous vector-valued functions, Banach function algebras, vectorvalued function algebras, characters, maximal ideals. 


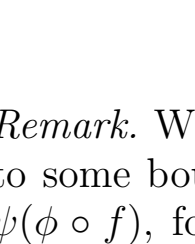

Banach J. Math. Anal. 10 (2016), no. 3, 608-620

http://dx.doi.org/10.1215/17358787-3607486

ISSN: $1735-8787$ (electronic)

http://projecteuclid.org/bjma

\title{
VECTOR-VALUED CHARACTERS ON VECTOR-VALUED FUNCTION ALGEBRAS
}

\author{
MORTAZA ABTAHI
}

Communicated by K. Jarosz

\begin{abstract}
Let $A$ be a commutative unital Banach algebra and let $X$ be a compact space. We study the class of $A$-valued function algebras on $X$ as subalgebras of $C(X, A)$ with certain properties. We introduce the notion of $A$-characters of an $A$-valued function algebra $\mathscr{A}$ as homomorphisms from $\mathscr{A}$ into $A$ that basically have the same properties as evaluation homomorphisms $\mathcal{E}_{x}: f \mapsto f(x)$, with $x \in X$. We show that, under certain conditions, there is a one-to-one correspondence between the set of $A$-characters of $\mathscr{A}$ and the set of characters of the function algebra $\mathfrak{A}=\mathscr{A} \cap C(X)$ of all scalar-valued functions in $\mathscr{A}$. For the so-called natural $A$-valued function algebras, such as $C(X, A)$ and $\operatorname{Lip}(X, A)$, we show that $\mathcal{E}_{x}(x \in X)$ are the only $A$-characters. Vector-valued characters are utilized to identify vector-valued spectra.
\end{abstract}

\section{INTRODUCTION AND PRELIMINARIES}

In this article, we consider only commutative unital Banach algebras over the complex field $\mathbb{C}$ (see [3], [4], [11], [18]).

Let $A$ be a commutative Banach algebra. The set of all characters of $A$ is denoted by $\mathfrak{M}(A)$. It is well known that $\mathfrak{M}(A)$, equipped with the Gelfand topology, is a compact Hausdorff space called the character space of $A$. For every $a \in A$, let $\hat{a}: \mathfrak{M}(A) \rightarrow \mathbb{C}, \phi \mapsto \phi(a)$, be the Gelfand transform of $a$. The algebra $A$ then can be seen, through its Gelfand representation $A \rightarrow C(\mathfrak{M}(A)), a \mapsto \hat{a}$, as a subalgebra of $C(\mathfrak{M}(A))$.

Copyright 2016 by the Tusi Mathematical Research Group.

Received May 23, 2015; Accepted Nov. 27, 2015.

2010 Mathematics Subject Classification. Primary 46J10; Secondary 46J20, 46H10, 46E40.

Keywords. Algebras of continuous vector-valued functions, Banach function algebras, vectorvalued function algebras, characters, maximal ideals. 


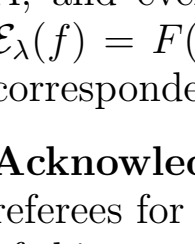

Banach J. Math. Anal. 10 (2016), no. 3, 608-620

http://dx.doi.org/10.1215/17358787-3607486

ISSN: $1735-8787$ (electronic)

http://projecteuclid.org/bjma

\title{
VECTOR-VALUED CHARACTERS ON VECTOR-VALUED FUNCTION ALGEBRAS
}

\author{
MORTAZA ABTAHI
}

Communicated by K. Jarosz

\begin{abstract}
Let $A$ be a commutative unital Banach algebra and let $X$ be a compact space. We study the class of $A$-valued function algebras on $X$ as subalgebras of $C(X, A)$ with certain properties. We introduce the notion of $A$-characters of an $A$-valued function algebra $\mathscr{A}$ as homomorphisms from $\mathscr{A}$ into $A$ that basically have the same properties as evaluation homomorphisms $\mathcal{E}_{x}: f \mapsto f(x)$, with $x \in X$. We show that, under certain conditions, there is a one-to-one correspondence between the set of $A$-characters of $\mathscr{A}$ and the set of characters of the function algebra $\mathfrak{A}=\mathscr{A} \cap C(X)$ of all scalar-valued functions in $\mathscr{A}$. For the so-called natural $A$-valued function algebras, such as $C(X, A)$ and $\operatorname{Lip}(X, A)$, we show that $\mathcal{E}_{x}(x \in X)$ are the only $A$-characters. Vector-valued characters are utilized to identify vector-valued spectra.
\end{abstract}

\section{INTRODUCTION AND PRELIMINARIES}

In this article, we consider only commutative unital Banach algebras over the complex field $\mathbb{C}$ (see [3], [4], [11], [18]).

Let $A$ be a commutative Banach algebra. The set of all characters of $A$ is denoted by $\mathfrak{M}(A)$. It is well known that $\mathfrak{M}(A)$, equipped with the Gelfand topology, is a compact Hausdorff space called the character space of $A$. For every $a \in A$, let $\hat{a}: \mathfrak{M}(A) \rightarrow \mathbb{C}, \phi \mapsto \phi(a)$, be the Gelfand transform of $a$. The algebra $A$ then can be seen, through its Gelfand representation $A \rightarrow C(\mathfrak{M}(A)), a \mapsto \hat{a}$, as a subalgebra of $C(\mathfrak{M}(A))$.

Copyright 2016 by the Tusi Mathematical Research Group.

Received May 23, 2015; Accepted Nov. 27, 2015.

2010 Mathematics Subject Classification. Primary 46J10; Secondary 46J20, 46H10, 46E40.

Keywords. Algebras of continuous vector-valued functions, Banach function algebras, vectorvalued function algebras, characters, maximal ideals. 


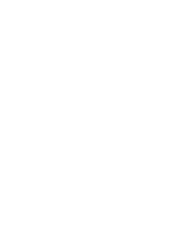

Banach J. Math. Anal. 10 (2016), no. 3, 608-620

http://dx.doi.org/10.1215/17358787-3607486

ISSN: $1735-8787$ (electronic)

http://projecteuclid.org/bjma

\title{
VECTOR-VALUED CHARACTERS ON VECTOR-VALUED FUNCTION ALGEBRAS
}

\author{
MORTAZA ABTAHI
}

Communicated by K. Jarosz

\begin{abstract}
Let $A$ be a commutative unital Banach algebra and let $X$ be a compact space. We study the class of $A$-valued function algebras on $X$ as subalgebras of $C(X, A)$ with certain properties. We introduce the notion of $A$-characters of an $A$-valued function algebra $\mathscr{A}$ as homomorphisms from $\mathscr{A}$ into $A$ that basically have the same properties as evaluation homomorphisms $\mathcal{E}_{x}: f \mapsto f(x)$, with $x \in X$. We show that, under certain conditions, there is a one-to-one correspondence between the set of $A$-characters of $\mathscr{A}$ and the set of characters of the function algebra $\mathfrak{A}=\mathscr{A} \cap C(X)$ of all scalar-valued functions in $\mathscr{A}$. For the so-called natural $A$-valued function algebras, such as $C(X, A)$ and $\operatorname{Lip}(X, A)$, we show that $\mathcal{E}_{x}(x \in X)$ are the only $A$-characters. Vector-valued characters are utilized to identify vector-valued spectra.
\end{abstract}

\section{INTRODUCTION AND PRELIMINARIES}

In this article, we consider only commutative unital Banach algebras over the complex field $\mathbb{C}$ (see [3], [4], [11], [18]).

Let $A$ be a commutative Banach algebra. The set of all characters of $A$ is denoted by $\mathfrak{M}(A)$. It is well known that $\mathfrak{M}(A)$, equipped with the Gelfand topology, is a compact Hausdorff space called the character space of $A$. For every $a \in A$, let $\hat{a}: \mathfrak{M}(A) \rightarrow \mathbb{C}, \phi \mapsto \phi(a)$, be the Gelfand transform of $a$. The algebra $A$ then can be seen, through its Gelfand representation $A \rightarrow C(\mathfrak{M}(A)), a \mapsto \hat{a}$, as a subalgebra of $C(\mathfrak{M}(A))$.

Copyright 2016 by the Tusi Mathematical Research Group.

Received May 23, 2015; Accepted Nov. 27, 2015.

2010 Mathematics Subject Classification. Primary 46J10; Secondary 46J20, 46H10, 46E40.

Keywords. Algebras of continuous vector-valued functions, Banach function algebras, vectorvalued function algebras, characters, maximal ideals. 